\title{
A New Method of structuring Hierarchies
}

\author{
Zhou Shipeng \\ Research Department 5 \\ No. 1 Beiyuan Da Yuan \\ An Ding Men Wai \\ Beijing 100012, CHINA

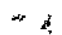 \\ Abstract
}

Structuring the hierarchy of a system is an important step, to solve problems with the Analytic Hierarchy Process. This paper gives and proves two theorems about the relationship between the hierarchy and the reachability matrix of the system. We propose a method of structuring hierarchy by directly using the reachability matrix of the system. This method is simpler and clearer than that of Interpretive Structural Modeling and is suitable for both manual calculating and computer processing. It fully shows the simplicity of the AHP.

\section{Introduction}

When we use the AHP to solvé a problem, the first thing to do is to structure the hierarchy for the problem. If the problem is fairly simple, there are only a few factors and their cross referencing and dominant relations are not difficult to deal with. Thus, there is no problem in structuring the hierarchy. ;However, if the problem involves many factors and their cross referencing is complicated, then it is difficult to obtain its hierarchy: by subjective, Iogical judgments.

In order to solve the above problems, we introduce mathematical models. At present, the most commonly method used is the Interpretive structural Modeling. This method involves many mathematical concepts making it rather difficult for those people whose mathematical bases are fairly poor to master it. For this reason, this paper proposes a method of structuring the hierarchy by directly using the reachability matrix of the system as the basis of Interpretive Structural Modeling. 


\section{The Method and Example}

\section{Reachability Matrix}

Assume $H$ to be a set in which the membership of elements is kmown. The membership of the set can be expressed by a binary matrix or a directed graph. Both methods of expression are equivalent. The order of the binary matrix is equal to the number of elements in the set. An arbitrary element $b_{i j}$ in the matrix is defined as follows

$$
\begin{aligned}
& 1 \text {, if } j \text { is subordinated to } i \\
& b_{i j}= \\
& 0 \text {, otherwise }
\end{aligned}
$$

and assume $b_{i j}=1$, namely, we assume that an element is subordinated to itself. So all the elements on the main diagonal for a binary matrix of an arbitrary order are equal to 1.

Definition 2.1: The product of two binary matrixes $A=\left(a_{i j}\right)$ and $B=\left(b_{i j}\right)$ is defined, as a binary matrix $c=\left(c_{i j}\right)$, given by:

$$
1 \text {, if } \sum_{k=1}^{n} a_{i k} b_{k j}>0
$$

$$
c_{i j}=
$$

$$
\text { 0, otherwise. }
$$

According to definition 2.1, for an arbitrary binary matrix $B$ there exists a minimum positive integer $\mathrm{k}$, for which:

$$
B^{k-1}<B^{k}=B^{k+1}
$$

where $B^{k-1}<B^{k}$ means that each element of $B^{k-1}$ is less than or equal to the corresponding element of $B^{k}$. $B^{k}$ is called the reachability matrix of the directed graph corresponding to $B$.

It can be easily seen that for a directed graph if we define its binary matrix $D=\left(d_{i j}\right)$ as follows: 
$I$ if element $i$ can reach $j$,

$d_{i j}=$

0 otherwise,

then $D$ is its reachability matrix.

The Method of Structuring The Hierarchy by The Reachability Matrix

Now we propose our method, the proof will be explained later.

The steps are as follows:

Step 1: Label the row and column of the reachability matrix $B$ of the system. The row and column corresponding to element $h_{i}$ in B are labeled $h_{i}$.

Step 2: Find out the columns of the reachability matrix B that are the same. If column $h_{i}$ and column $h_{j}$ are the same, then elements $h_{i}$ and $h_{j}$ belong to the same level, and the row $h_{i}$ and column $h_{i}$ are labelled $h_{i, j}$. Delete row $h_{j}$ and column $h_{j}$. Repeat this process until there exist no two columns that are the same. Denote the new reachability matrix by $B^{\prime}$.

Step 3: Find those columns that have only one $I$ in the reachability matrix $B^{\prime}$. The elements assigned to the labels of these columns belong to the same level.

Step 4: In the reachability matrix cross off those/ rows and columns corresponding to the labels that are found in step 3 . Repeat step 4 until there no columns that has only one 1 . An Example

Now we cite an example in illustration of the above method. The binary matrix of Figure 2.1 is given by: 


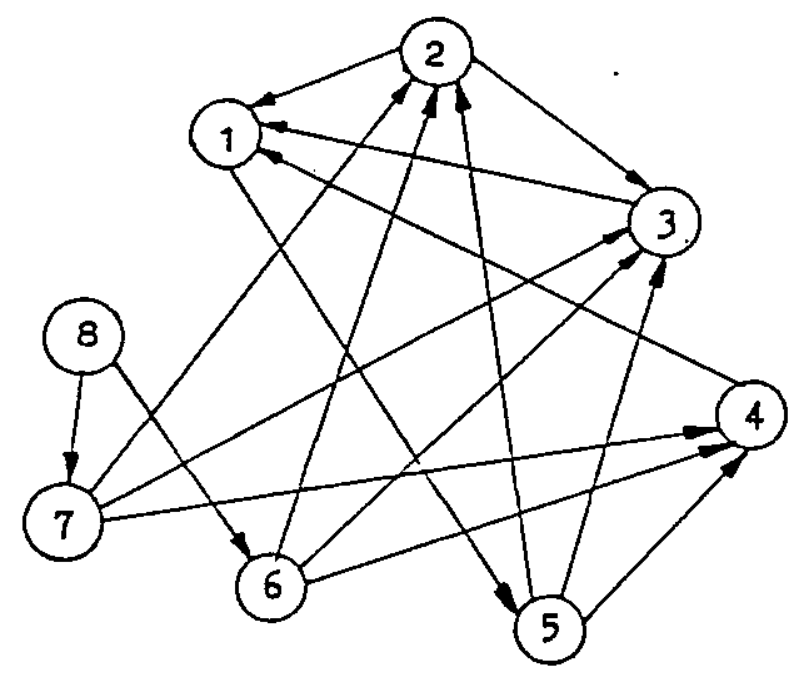

Figure 2.1. An example

$$
B=\left[\begin{array}{llllllll}
1 & 0 & 0 & 0 & 1 & 0 & 0 & 0 \\
1 & 1 & 1 & 0 & 0 & 0 & 0 & 0 \\
1 & 0 & 1 & 0 & 0 & 0 & 0 & 0 \\
1 & 0 & 0 & 1 & 0 & 0 & 0 & 0 \\
0 & 1 & 1 & 1 & 1 & 0 & 0 & 0 \\
0 & 1 & 1 & 1 & 0 & 1 & 0 & 0 \\
0 & 1 & 1 & 1 & 0 & 0 & 1 & 0 \\
0 & 0 & 0 & 0 & 0 & 1 & 1 & 1
\end{array}\right]
$$

and its reachability matrix is given by:

$$
B^{4}=\left[\begin{array}{llllllll}
1 & 1 & 1 & 1 & 1 & 0 & 0 & 0 \\
1 & 1 & 1 & 1 & 1 & 0 & 0 & 0 \\
1 & 1 & 1 & 1 & 1 & 0 & 0 & 0 \\
1 & 1 & 1 & 1 & 1 & 0 & 0 & 0 \\
1 & 1 & 1 & 1 & 1 & 1 & 0 & 0 \\
1 & 1 & 1 & 1 & 1 & 1 & 1 & 1
\end{array}\right]
$$

According to step 1 and step 2 of the method, label and relabel the reachability matrix as follows: 


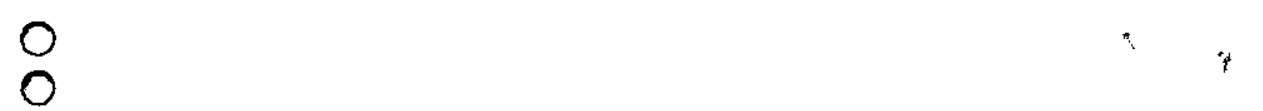

$$
\begin{aligned}
& \begin{array}{lllllllll}
h_{1} & h_{2} & h_{3} & h_{4} & h_{5} & h_{6} & h_{7} & h_{8}
\end{array}
\end{aligned}
$$

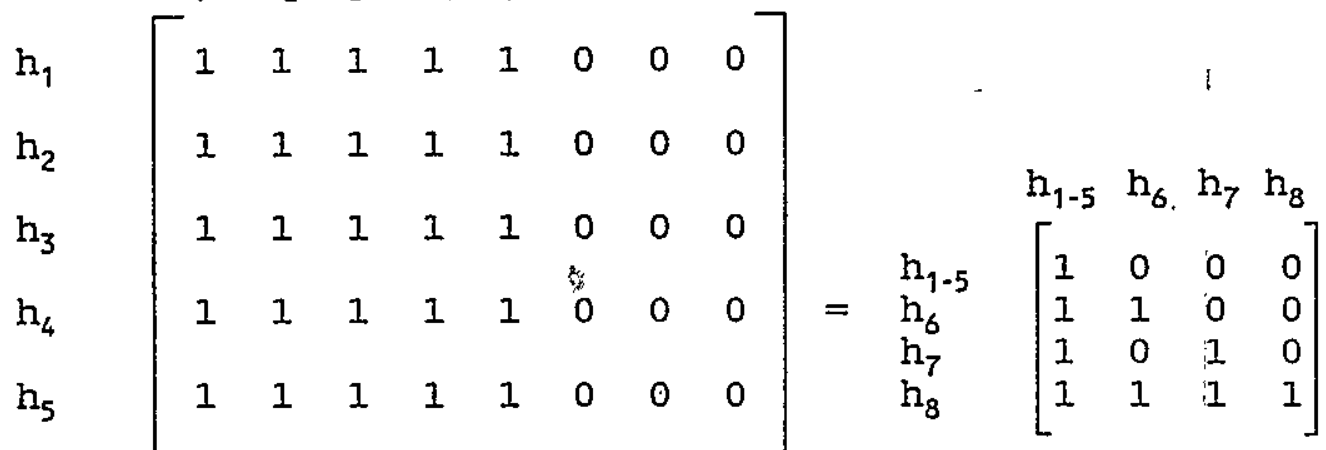

$$
\begin{aligned}
& h_{6} h_{7} \quad\left[\begin{array}{llllllll}
1 & 1 & 1 & 1 & 1 & 1 & 0 & 0 \\
1 & 1 & 1 & 1 & 1 & 0 & 1 & 0 \\
1 & 1 & 1 & 1 & 1 & 1 & 1 & 1
\end{array}\right]
\end{aligned}
$$

According to step 3, from the above reachability matrix, only Column $h_{8}$ has only one 1 , so $L_{1}={ }^{*}\left\{h_{8}\right\}$. Cross off Row $h_{9}$ and column $h_{8}$, then we get the following matrix

$$
\begin{aligned}
& h_{1,2,3,4,5} \\
& h_{1,2,3,4,5} \\
& h_{6} \\
& h_{7}
\end{aligned}\left[\begin{array}{lll}
1 & 0 & 0 \\
1 & 1 & 0 \\
1 & 0 & 1
\end{array}\right]
$$

Because both column $h_{6}$ and column $h_{7}$ have only one $I, I_{2}=\left\{h_{6}, h_{7}\right\}$. We now delete Rows $h_{6}$ and $h_{7}$, and columns $h_{6}$ and $h_{7}$. Then we get. $I_{3}$ $=\left\{h_{1,2,3,4,5}\right\}=\left\{h_{1}, h_{2}, h_{3}, h_{4}, h_{5}\right\}$. Figure 2.2 shows the resulting hierárchy.

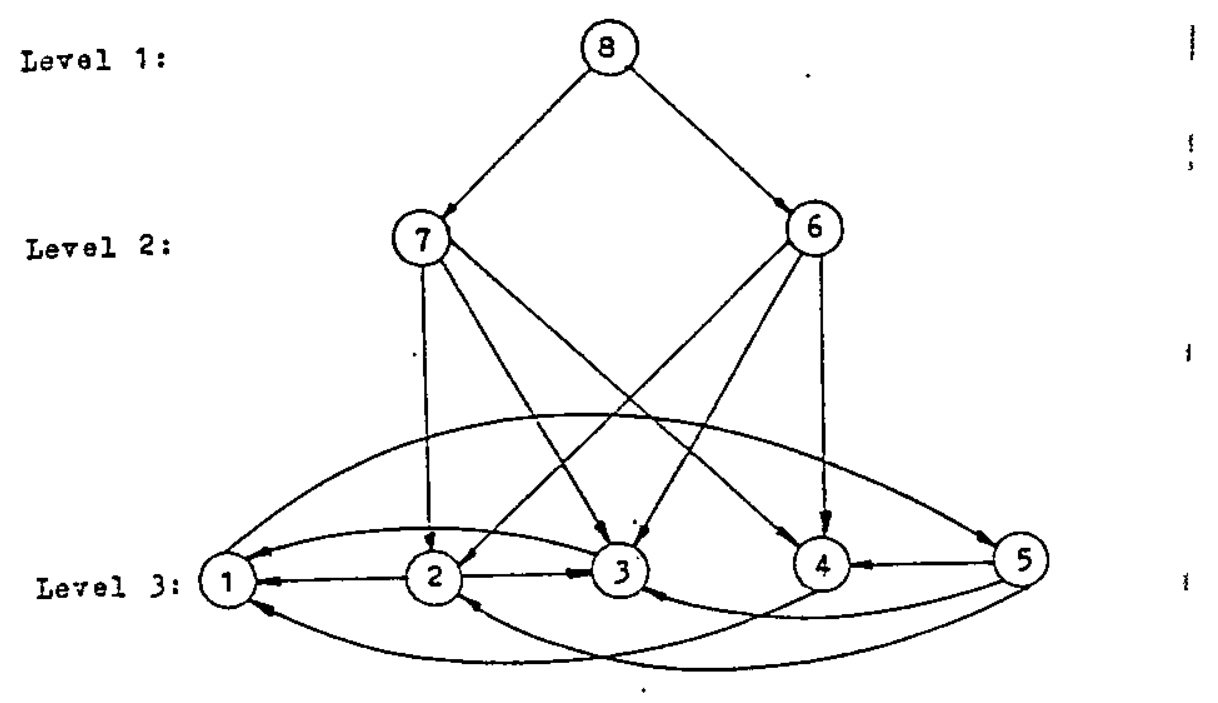

Figure 2.2. Resulting Hierarchy 


\section{Proof of the Method}

Definition 3.1: An element $h_{j}$ is said to be accessible from element $h_{i}$, if there exists a path from $h_{i}$ to $h_{j}$ in the directed graph.

Definition 3.2: An element $h_{j}$ is said to be the antecedent of $h_{j}$ if $h_{i}$ is accessible from $h_{j}$.

Let $R\left(h_{i}\right)$ be the set of all accessible elements from $h_{i}$ in $H$, let $A\left(h_{i}\right)$ be the set of the antecedents of $h_{i}$, and let $B^{k}=\left(b_{i j}\right)$ be the reachability matrix of $H$. Then

$$
\begin{aligned}
& R\left(h_{i}\right)=\left\{h_{j} \in H \mid b_{i j}=1\right\} \\
& A\left(h_{j}\right)=\left\{h_{j} \in H \mid b_{j i}=1\right\} .
\end{aligned}
$$

Thus, the element $h_{i} \in H$ belongs to Level $I_{1}$ (the highest level) if and only if:

$$
A\left(h_{i}\right)=A\left(h_{i}\right) \cap R\left(h_{i}\right)
$$

Definition 3.3: The hierarchy ${ }^{-} \mathrm{H}$ is said to be interior independent if for an arbitrary $h_{i} \in H$,

$$
A\left(h_{i}\right) \cap R\left(h_{i}\right)=\left\{h_{i}\right\}
$$

Lemma 1: Let $H \equiv\left\{h_{i}, h_{2}, \ldots, h_{n}\right\}$ be a set in which the membership of the elements is determined, and let $B$ to be its reachability matrix. Then the following three propositions are equivalent:

(i) The elements $h_{i}$ and $h_{j}$ are antecedents of each other.

(ii) The columns corresponding to $h_{i}$ and $h_{j}$ in $B$ are the same.

(iii) The Rows $h_{\mathfrak{i}}$ and $h_{j}$ in $B$ are the same.

Proof: First we prove that if (i) hold then (ii) also holds. Since the elements $h_{i}$ and $h_{j}$ are the antecedents of each other, then, we have

$$
h_{i} \in A\left(h_{j}\right), \quad h_{j} \in A\left(h_{i}\right)
$$

According to Definition 3.2 and (3.2), we have:

$$
A\left(h_{i}\right) \subset A\left(h_{j}\right) \quad A\left(h_{j}\right) \subset A\left(h_{i}\right)
$$

and hence, $A\left(h_{j}\right)=A\left(h_{j}\right)$, namely, the columns $h_{i}$ and $h_{j}$ in $B$ are the same.

Second, we prove that if (ii) holds, then (iii) also holds. If the columns $h_{i}$ and $h_{j}$ in $B$ are the same; then, $A\left(h_{j}\right)=A\left(h_{j}\right)$. According to (3.2), we have (3.5), and according to ( 3.1$)$, we have: 


$$
h_{j} \in R\left(h_{i}\right), \quad h_{i} \in R\left(h_{j}\right)
$$

Therefore, we have:

$$
R\left(h_{j}\right) \subset R\left(h_{i}\right), R\left(h_{i}\right) \subset R\left(h_{j}\right)
$$

and hence, $R\left(h_{i}\right)=R\left(h_{j}\right)$, and the rows $h_{i}$ and $h_{j}$ are the are identical.

Finally, we prove that if (iii) holds, then (i) holds. Since the rows $h_{i}$ and $h_{j}$ are the same, that is, $R\left(h_{i}\right)=R\left(h_{j}\right)$, we have (3.7). According to (3.2), we have:

$$
h_{i} \in A\left(h_{j}\right), h_{j} \in A\left(h_{i}\right)
$$

and hence, the elements $h_{i}$ and $h_{j}$ are antecedents of each, other.

Theorem 1: A necessary and sufficient condition for a hierarchy to be interior independent is that its reachability matrix does not have any two identical columns;

Proof: (Necessity) Assume that the hierarchy is interior dependent and that that there exist two columns in the reachability matrix that are identical, e.g., $b_{h i, k}=b_{h j, k}$, for all k. By Lemma 1 , we have:

$$
h_{i} \in A\left(h_{j}\right), \quad h_{j} \in A\left(h_{i}\right)
$$

and

$$
h_{i} \in R\left(h_{j}\right), \quad h_{j} \in R\left(h_{i}\right)
$$

and hence,

$$
\left\{h_{i}, h_{j}\right\} \in A\left(h_{i}\right) \cap R\left(h_{j}\right)
$$

which contradicts the assumption that the hierarchy is interior independent. So there do not exist two columns in the reachability matrix that are identical.

(Sufficiency) If there are no two columns identical, then by Lemma 1 , two distinct elements cannot be antecedents of each other and hence, the hierarchy must be interior independent.

Corollary 1: A hierarchy is interior dependent if and only if there exists at least two identical columns in its reachability matrix.

corollary 2: If the columns $h_{j}$ and $h_{j}$ of the reachability matrix are the same, then they belong to the same level in the hierarchy.

For an interior dependent hierarchy $\mathrm{H}$, by Corollary 1 , there exists at least two identical columns in its reachability matrix $B$. Let columns $h_{i}$ and $h_{j}$ be those columns. According to Lemma 1 , not 
only row $h_{j}$ and row $h_{j}$ are the same, but also the elements $h_{i}$ and $h_{j}$ are antecedents of each other, and by corollary 2 , the elements $h_{i}$ and $h_{j}$ belong to the same level. Therefore, we can take element $h_{i}$ and element $h_{j}$ as a system element and delete the column $h_{j}$ and the row $h_{j}$. Following these steps can translate the interior dependent hierarchy into an interior independent hierarchy. This is the theoretical basis for step 2 of the method. The following theorem provides the theoretical basis for structuring the interior independent hierarchy or step 3 of the method.

Theorem 2: Let $\mathrm{H}$ be an interior independent hierarchy, and let. $\mathrm{B}$ be its reachability matrix. An element $h_{i}$ in $H$ belongs to the highest level of $H$ if and only if the column $h_{i}$ in $B$ has only one entry equal to 1 .

Proof: Follows from Lemma 1 and Theorem 1 .

\section{REFERENCES}

Saaty, T.L., 1980, "The Analytic Hierarchy Process," McGrawHill International Book Company.

Xu Shuo, 1988, "The Analytic Hierarchy Process," Tianjin University Press, Tianjin. 